\title{
Lessons learned from a table-top exercise on monitoring a large-scale cross-border contamination in the aftermath of a nuclear accident
}

\author{
B. Zorko ${ }^{1}$, J. Camps ${ }^{2}$ and T. Petrovič ${ }^{1}$ \\ 1 Jozef Stefan Institute, Jamova cesta 39, 1000 Ljubljana, Slovenia. \\ 2 SCK•CEN, Belgian Nuclear Research Centre, Boeretang 200, 2400 Mol, Belgium.
}

\begin{abstract}
A table-top exercise on 'Monitoring a large-scale cross-border contamination in the aftermath of a nuclear accident' was organized within the FP7-project PREPARE with the focus on the long-term emergency monitoring including transboundary and transnational aspects. From the detailed and content-rich answers from all participants of the table-top exercise, it can be concluded that competent persons with a lot of practical experience in the field were present to play the exercise. Answers were very useful because they were based on the experience from the real situation (e.g. Fukushima accident). It seems that the sampling procedures and measurement strategies are well defined. It was found out that all available communication channels can be established among the crisis centre, teams on the field and other institutions and/or teams involved (e.g. fire brigades, police, army fixed laboratories). From the answers, it comes out that some activities in the emergency are not completely harmonized transnationally, especially dispersion modelling, exchange of data, etc. The majority of the teams claimed that RODOS platform can be applied as the primary tool, but also other dispersion models, in-country developed, would be used in the nuclear emergency. From the answers, it stems out that numbering of specialized teams on the ground and the measurement capacities either in mobile laboratories or fixed laboratories should be concerned in small countries with nuclear installations, and even more pronounced in countries without Nuclear Power Plants (NPPs) on their territories.
\end{abstract}

Keywords: table-top exercise / transnational and transboundary impact / interactive web platform

\section{Introduction}

In the territories of European Union (EU) member states, the radioactive contamination threat comes from nuclear accidents in nuclear facilities and the radiological dirty bomb following the global terrorism, illegal transport of radioactive material as well as smuggling should be concerned substantially.

In nuclear accident or radiological emergency, the radioactive material is emitted and dispersed into the environment following characteristic transfer pathways. The protection and rescue measures to be deployed in such an event are laid down in national emergency response plans. Whilst the emergency monitoring strategy details cannot be planned in advance, greater efficiency can be achieved, if planning in terms of coordination, access to resources, outline strategy, priorities and other matters are included as part of the emergency planning process (IAEA, 2015). The adequacy of nuclear and radiological emergency response arrangements can be evaluated through the audit and review of plans, procedures and infrastructure. The ability to carry out the required emergency actions can be assessed through audits and reviews of past performance, but it is most commonly evaluated through exercises. Thus, the exercises represent a powerful tool for verifying and improving the quality of emergency response arrangements. Among various types of exercises, a table-top exercise, which is a discussion-type exercise conducted around a table, plays a highest importance in the emergency preparedness management.

To contribute to the field of the radiological emergency preparedness, the table-top exercise on 'Monitoring a largescale cross-border contamination in the aftermath of a nuclear accident' was organized within the FP7-project PREPARE. The main goal of this table-top exercise was to disseminate the existing emergency preparedness plans for emergency monitoring on a large-scale contamination, based on a hypothetical accident scenario affecting different countries and identify possible pitfalls.

\section{Methods}

The table-top was organized by JSI, Slovenia, in collaboration with SCK•CEN, Belgium in Ljubljana, Slovenia on 11 and 12 December 2013 (Zorko and Camps, 2016). The table-top exercise was attended by 28 participants from 15 different European countries. The exercise was based on a credible but hypothetical scenario. The fictive-affected nuclear site was placed in a complex terrain near the border of three countries. It was supposed that the radioactive contamination affected parts of all three countries. With this approach, we simulated and tested different responses in the case of the 
transboundary impact and of course exchange of information and data. Some data used in the exercise were prepared in advance (source term, dose-rate and ground deposition contamination maps; they were prepared using the ATSTEP dispersion model integrated into the decision support system RODOS (Päsler-Sauer, 2007)). The scenario was conducted not real time with certain time leaps to cover an extended time in the aftermath of the severe accident. To simulate and to test different responses in the case of a transboundary impact, the participants were divided into three groups.

The scope of the exercise was to discuss, elaborate and evaluate the emergency preparedness plans related to largescale contamination emergency monitoring, based on a severe nuclear emergency with serious transnational and transboundary implications. The objectives of the exercise were to identify and discuss different national and/or regional emergency monitoring strategies in case of a large-scale nuclear accident with transboundary impact, including priorities, resources, good practices, sampling and measurement strategies, measurement capacity, ways to exchange monitoring data and interpretation of modelled and raw data from the field measurements.

The exercise was played interactively on a web-based platform which was developed especially for this table-top exercise. A very flexible web-based tool was enabled to insert at regular intervals new scenario information including questions for the players (injects) on monitoring strategy and to collect all information and actions from the players. It is also served as a repository to drop larger files which helped players to answer their injects thoroughly. Moreover, it also served as an evaluation tool - providing the full list of answers from all players (database).

\section{Results and discussion}

When teams played the exercise, they always used the protocols and procedures from national emergency response plans. It was found out that the competent authorities in general manage the emergency monitoring strategies, recovery strategies and dose assessments (the top-down hierarchy), except in UK. It was found out that the activation of the resources and the entities from particular national organization structures are carried in various ways, depending on the information collected in the initial stage. It is fairly common that the site operator would inform a number of organizations according to its notification arrangements. Thus, the emergency monitoring strategy was regularly reviewed to take account of the changing situation as more information became available of the developing scenario, positively challenged by contamination maps produced by dispersion modelling. From the answers of the participant, it is significant that dispersion modelling is a primary tool in the initial decisionmaking, preparing viable emergency strategy, taking countermeasures in place and planning further activities related to evolution of the nuclear emergency. Data from the monitoring network, when available, will be used to verify model predictions. The field-assistance teams will be asked to confirm the dispersion model data by providing measurements on the ground, and they will be directed to move away from the plume. Input from the early warning network is used constantly in order to confirm the plume dispersion. These data are collected from the environmental radioactivity group and passed to the radiation protection group as a feedback for their calculations. The aerial survey team should be ready to perform measurements few hours from the beginning of the event. A first contamination map (dose-rate or ground fall-out) of possible release is drawn even at the early stage. Those two activities are not common, but highly desirable.

All teams played the scenario with well-defined and wellprepared sampling strategies (IAEA, 1999). By many teams, the sampling is managed by means of contamination maps produced on the basis of the data from aerial survey using either helicopters or aircrafts (France, Czech Republic, UK, Switzerland, Belgium, Finland, Germany and Portugal). Besides vehicles equipped for sampling and car-borne measurements, which are standard for all teams presented at the exercise, the Finnish colleagues introduced a special sampling strategy using VASSIKA backpacks.

In the early stage (facility danger, plume passage) of an accident the direct gamma ray dose rate, air-sampling using aerosol and iodine (charcoal) filters, rainwater and ground deposition (in combination with dispersion modelling forecasting) would take place, while later on (when discharge stops or after the transverse of a radioactive cloud), sampling would rather lean on locally produced foodstuff (meat, vegetables, milk, other farm products, drinking water, etc.).

The measurement strategy should follow well-prepared sampling strategy. Some teams have expressed concern that the measurement capacity either in mobile laboratories (field assistance teams (FAT)) or fixed laboratories might be insufficient as the samples would pile-up.

From the answers of the participants, it comes out that in the first stage of the scenario, the activity concentrations of ${ }^{131} \mathrm{I},{ }^{58} \mathrm{Co}$ and ${ }^{60} \mathrm{Co}$ would be of a main concern, while later on, the activity concentrations or specific activities of ${ }^{134} \mathrm{Cs},{ }^{137} \mathrm{Cs}$, ${ }^{3} \mathrm{H}$ and ${ }^{90} \mathrm{Sr}$ would be of utmost concern. Some teams reported also alpha spectrometric measurements.

The mobile laboratories are well equipped with $\mathrm{HPGe}, \mathrm{NaI}$ and $\mathrm{LaBr}_{3} \mathrm{Ce}$ spectrometers in a combination with hand-held and other relevant devices, while fixed laboratories can perform high sensitivity and radiochemical measurements.

The participant identified several ways to exchange measured data (IAEA, 2012). Almost all countries described their own web-based communication tools in case of a nuclear emergency. These systems connect major national stakeholders. Those national on-line tools provide immediate, direct and secure (independent network) information exchange (messages and attachments) between all national stakeholders at the same time. Most of the teams described their national web tools very multifunctional and reliable, but no explicit data formats were discussed in detail. Evidently, these data also feed the EURDEP database automatically. All would be transmitted via ECURIE (early notification system is the practical implementation of the Council Decision 87/600) and IAEA USIE (EMERCON/ENATOM) web platforms.

Besides the established communication channels, it is also important and interesting how those data exchange are transmitted. For example, the team France presented that dose rate data are transmitted from the field to the crisis centre via satellite transmission, which might be an enhanced and powerful alternative to the global transmission routes 
established on the ground routes (mobile data service, telephone line, etc.).

\section{Conclusions}

The findings of the table-top exercise give a good foundation to study the emergency monitoring activities thoroughly in details. This type of exercise was played for the first time, and the exercise participants recognized it as an important approach to challenge the difficulties during emergency monitoring on a large scale with transboundary and transnational impact. The pronounced advantage of this exercise was that a special webbased platform was developed. It was found flexible, generic, what allows its further development, especially in case-oriented exercises and studies. It also provides a secure way of exchange of information, meaning that exercises using this web platform could be played from home, not necessary for the participants (players) to travel.

The data processing between the field assistance team and the crisis centre would likely be a big challenge still, since pictorial data (contamination maps) would be of non-standardized formats, and it could potentially block some activities due to bad resolution or language specificity. Besides, different competent authorities rely on various dispersion models which outputs are also not standardized. So, we come across with double-fold challenge related to handling the pictorial data.

From the answers, we found out that generally the activation time in crisis centre is very short from $0.5 \mathrm{~h}$ to a maximum of $2 \mathrm{~h}$. Contrary, it was not identified the time needed for FAT to reach a given location nor what time they would need to be either on stand-by and ready to start their activities on the ground. This is a factor that might be challenged in future, if such an exercise would be laid down again.

It seems that most of the players were focused on the population area and the nearby food producing area, while forests and mountain region were not of primary interest. This kind of giving priorities in sampling is not surprising, and it is not in contradiction with several findings in the past related to the Chernobyl accident. Most of the hot spots or elevatedspecific activities were find in remote places, forests, etc.
If the exercise is to be repeated at whatever format, it would be recommended that each national team would have more than one person (preferably three). The reason lies in the complexity of national response protocols which should be coordinated among several entities, and subsequent activities could sometimes be unclear and wait to be managed properly.

The findings of the exercise that almost all countries would have problems with human resources and measurement capacities after some time are serious, but these kinds of problems cannot be solved or challenged with this kind of exercises. This is mainly related to available national resources.

It seems that national nuclear emergency plans have been prepared for the events which occur on the homeland territory, and to some extent, they underestimate the possibility of transboundary impact. Several teams asked for the help from the RANET (IAEA), some of them expressed willingness to accept it, but during the exercise, they haven't explicitly asked for it. Some teams were reserved, so the capacity of the response teams and the capacity of laboratories are still a challenging factor.

Acknowledgement. The research leading to these results has received funding from the European Atomic Energy Community Seventh Framework Programme FP7/2012-2013 under grant agreement 323287.

\section{References}

IAEA (1999) IAEA-TECDOC-1092 Generic Procedures for Monitoring in a Nuclear or Radiological Emergency.

IAEA (2012) EPR-IEComm, Operations Manual for Incident and Emergency Communication.

IAEA (2015) Series No. GSR Part 7, Preparedness and Response for a Nuclear or Radiological Emergency.

Päsler-Sauer J. (2007) Description of the Atmospheric Dispersion Model ATSTEP. Available at: resy5.iket.kit.edu/RODOS/Docu ments/Public/HandbookV6f/Volume3/ATSTEPfinal2.pdf.

Zorko B., Camps J. (2016) Monitoring a Large Scale Cross Border Contamination in the Aftermath of a Nuclear Accident, Deliverable number: D7.6.

Cite this article as: B. Zorko, J. Camps, T. Petrovič. Lessons learned from a table-top exercise on monitoring a large-scale cross-border contamination in the aftermath of a nuclear accident. Radioprotection 51(HS2), S175-S177 (2016). 